\title{
ENGEVISTA
}

Página da revista: http://www.uff.br/engevista/seer/

\section{Composição da fibra da casca de coco verde in natura e após pré-tratamentos químicos}

\author{
Mirelle Márcio Santos Cabral ${ }^{1}$ \\ Ana Karla de Souza Abud ${ }^{2}$ \\ Martha Suzana Rodrigues dos Santos Rocha ${ }^{3}$ \\ Renata Maria Rosas Garcia Almeida ${ }^{4}$ \\ Márcia Andréa Gomes ${ }^{5}$
}

\begin{abstract}
Resumo: O aumento do consumo da água de coco leva a uma maior geração de resíduos, impactando em problemas ambientais e merecendo atenção de pesquisadores para o aproveitamento desta biomassa. Este trabalho avalia a composição da fibra da casca de coco verde, na forma in natura e após pré-tratamento ácido e alcalino, investigando as características lignocelulósicas desta biomassa como mais uma alternativa para o desenvolvimento e produção do etanol. Observou-se elevada concentração de lignina (40,10\%), proporcionando alto grau de durabilidade e resistência ao material, e significativa quantidade de celulose $(24,70 \%)$, oferecendo boas condições para sua utilização na produção de etanol de segunda geração. O pré-tratamento alcalino, apesar da significativa perda em celulose, apresentou maior solubilização de lignina (80\%), tornando-se o mais viável para estudos da produção de etanol 2G.
\end{abstract}

Palavras-chave: Aproveitamento, casca de coco, celulose, pré-tratamento.

\footnotetext{
${ }^{1}$ UFAL - Universidade Federal de Alagoas

2 UFS - Universidade Federal de Sergipe

${ }^{3}$ UFSCar - Universidade Federal de São Carlos

${ }^{4}$ UFAL - Universidade Federal de Alagoas

${ }^{5}$ UFAL - Universidade Federal de Alagoas
} 
ISSN: $1415-7314$

ISSN online: $2317-6717$

Abstract: The increase of green coconut water consumption leads to a high volumes of waste generation, impacting an environmental problems and deserving attention of researchers to use of this biomass. This study evaluates the coconut shell fiber composition, in dried form and after acid and alkaline pretreatments, investigating the lignocellulosic characteristics of this biomass as an alternative for the development and ethanol production. It was observed a high lignin content (40.10\%), providing a high degree of durability and strength to the material, and significant amount of cellulose (24.70\%), offering good conditions for their use in 2nd generation ethanol production. The alkaline pretreatment, despite the significant loss of cellulose, showed more soluble lignin (80\%), making it more feasible for the studies of $2 \mathrm{G}$ ethanol production.

Keywords: Use, coconut shell, cellulose, pretreatment. 


\section{Introdução}

O uso da biomassa viabiliza o desenvolvimento de novas tecnologias, a exemplo dos biocombustíveis, tornando-se fator fundamental para a redução dos impactos econômicos (diversificação da matriz energética, diversificação da agricultura, aumento de investimentos na pesquisa e sustentabilidade) e ambientais (redução de ar poluente, sequestro de carbono, redução de gases do efeito estufa, melhoria da terra e uso adequado da água) (Ballat, 2011; Chemmés et al., 2013).

Os materiais lignocelulósicos são polímeros de carboidratos complexos, onde $90 \%$ da massa seca são, basicamente, compostas por celulose $\left(\mathrm{C}_{6} \mathrm{H}_{10} \mathrm{O}_{5}\right)_{\mathrm{x}}$, hemicelulose $\left(\mathrm{C}_{5} \mathrm{H}_{8} \mathrm{O}_{4}\right)_{\mathrm{m}}$ e lignina $\left(\mathrm{C}_{9} \mathrm{H}_{10} \mathrm{O}_{3}\left(\mathrm{OCH}_{3}\right)_{0,9-1,7}\right)_{\mathrm{n}}$, sendo o restante $(10 \%)$ extrativos e cinzas (Balat, 2011). A composição química do material é fator crucial na produção de biocombustível durante o processo de bioconversão.

Entre os biocombustíveis, destaca-se a produção de etanol de segunda geração, tendo a etapa de pré-tratamento da biomassa como processo mais desafiador, onde se visa separar a matriz de lignina, reduzir a cristalinidade da celulose, aumentando sua fração amorfa, e solubilizar a hemicelulose, tornando o hidrolisado da celulose mais acessível às hidrólises biológicas e químicas (Sarkar et al., 2012). Para ser considerado eficaz, o pré-tratamento precisa disponibilizar grandes quantidades de açúcares fermentáveis, evitar perdas, degradação dos carboidratos, formação de subprodutos inibidores para os processos de hidrólise e fermentação, bem como ser viável economicamente. Atualmente, os métodos de pré-tratamento consistem em processos físicos, químicos ou físico-químicos (Barreto, 2009).

O Brasil é o quarto maior produtor mundial de cocos, com produção aproximada de 2,8 milhões de toneladas em uma área de 257 mil hectares, onde 15\% desta produção é estimada para o mercado do coco verde (Senhoras, 2003; Martins e Jesus Jr, 2014). O crescente consumo da água de coco (100 a 350 milhões de litros/ano), impulsionado pela inclusão de hábitos saudáveis no comportamento da população brasileira, traz consigo problemas quanto ao que se deve fazer com o subproduto (cascas), que constituem 80 a $85 \%$ do peso bruto do fruto. Normalmente depositadas em lixões a céu aberto, vazadouros, encostas e aterros sanitários, além de degradarem a paisagem e produzirem mau cheiro, contribuindo para a transmissão de doenças, transformam-se em um sério problema ambiental ao encontrar condições anaeróbias para a produção de gás metano, um dos gases mais importantes do efeito estufa (Rosa, 1998; Brito et al., 2004; Veloso et al., 2013). Apesar de orgânico, o resíduo do coco é um material de difícil decomposição, demorando mais de 8 anos para se decompor completamente (Carrijo et al., 2002).

De forma a agregar valor, evitando/reduzindo o efeito negativo desse resíduo no ambiente, várias alternativas têm sido propostas, a exemplo de sua utilização na agricultura, na forma de substratos para a produção de mudas (Carrijo et al., 2002), sendo a casca de coco verde viável para

ENGEVISTA, V. 19, n.1 , p. 99-108, Janeiro 2017. 
essa aplicação por suas fibras serem quase inertes e terem alta porosidade, facilidade de produção, baixo custo e alta disponibilidade (Sampaio et al., 2008). Também, para produção de briquetes, contribuindo para a preservação da vegetação nativa, pela substituição à lenha (César et al., 2010; Pimenta et al., 2015), produção de enzimas, onde o pó da casca de coco verde se revelou ótimo indutor na produção de celulases (Maciel et al., 2010), adsorção de metais pesados (Silva et al., 2013), isolamento acústico, com a fibra misturada ao aglomerado de cortiça expandido (Senhoras, 2004), na indústria automobilística, substituindo a espuma derivada do petróleo (Costa e Gebara, 2001) e na construção civil, em pranchas pré-moldadas ou em fibro-cimento (Pereira et al., 2013).

Este trabalho analisa a composição da fibra da casca de coco verde in natura e após prétratamento ácido e alcalino, avaliando a viabilidade de uso deste resíduo na produção de etanol 2G.

\section{Metodologia}

A casca do coco verde foi obtida de vendedores de água de coco na própria Universidade Federal de Alagoas, os quais descartam este resíduo após a coleta da água. As mesmas foram levadas ao Laboratório de Ensino em Engenharia Química, dilaceradas em tamanhos menores, sanitizadas em hipoclorito de sódio $100 \mathrm{ppm}$ por 15 min e submetidas à secagem $\left(50^{\circ} \mathrm{C}\right)$ até peso constante. Em seguida, foram trituradas em forrageira elétrica e, posteriormente, em liquidificador, sendo separados pó e fibra da casca de coco após passagem por peneira.

No processo de pré-tratamento, aproximadamente $5 \mathrm{~g}$ de amostra de fibra da casca do coco verde foram misturadas a $100 \mathrm{~mL}$ de solução (ácido sulfúrico $5 \%$ ou hidróxido de sódio $5 \%$ ), em frascos Erlenmeyers de $500 \mathrm{~mL}$, e autoclavadas a $121^{\circ} \mathrm{C}$ e 1 atm por $40 \mathrm{~min}$. Após o período de reação e despressurização da autoclave, ocorreu a separação das frações líquida e sólida por filtração. Do líquido, foram analisadas a quantidade de açúcares liberados, medido em ART (açúcares redutores totais). A parte sólida foi lavada com água destilada à temperatura ambiente até que o líquido residual chegasse a $\mathrm{pH}$ neutro, sendo o resíduo, então, colocado para secagem em estufa à 50 ${ }^{\circ} \mathrm{C}$ até massa constante.

As fibras da casca do coco foram caracterizadas de acordo com procedimento descrito por Gouveia et al. (2009), obtendo-se a composição química quanto aos teores de celulose, hemicelulose e lignina. Todas as amostras (in natura e pré-tratada) foram caracterizadas realizando-se uma hidrólise ácida. Antes da caracterização, determinou-se a quantidade de extrativos, onde em um sistema de extração Soxhlet foi colocado um cartucho com $4 \mathrm{~g}$ (massa seca) de fibra da casca de coco verde, acoplando-se a ele um balão de fundo redondo contendo $190 \mathrm{~mL}$ de etanol 95\%, o qual ficou sobre refluxo por 12 horas, num ciclo mínimo de 6 sifões por hora. O líquido contendo os extrativos foi colocado em rotaevaporador até se obter um líquido viscoso, que foi retirado com um pouco de etanol e colocado em uma placa de Petri previamente tarada, deixando-a na capela de exaustão ligada até se obter massa constante. 
Para a hidrólise ácida, $1 \mathrm{~g}$ de fibra da casca do coco verde, devidamente moída em liquidificador industrial foi transferida para béqueres de $100 \mathrm{~mL}$, sofrendo tratamento com $10 \mathrm{~mL}$ de $\mathrm{H}_{2} \mathrm{SO}_{4}$ à $72 \%$ (v/v), sob vigorosa agitação, em um banho termostatizado a $45{ }^{\circ} \mathrm{C}$ por $8 \mathrm{~min}$. As amostras, transferidas para Erlenmeyers de $500 \mathrm{~mL}$, com inserção de $275 \mathrm{~mL}$ de água destilada, foram autoclavadas por $30 \mathrm{~min}$ a $120^{\circ} \mathrm{C}$. Após a descompressão da autoclave, os frascos foram retirados e resfriados à temperatura ambiente, sendo a fração sólida separada da fração líquida por filtração em papel de filtro qualitativo. A fração líquida foi transferida para balão volumétrico de 500 $\mathrm{mL}$, tendo o seu volume posteriormente completado com água destilada. A solução foi armazenada para análises posteriores de carboidratos, ácidos orgânicos, furfural, hidroximetilfurfural (HMF) e lignina solúvel.

Para a determinação de lignina insolúvel, o material retido no papel de filtro foi lavado com $1500 \mathrm{~mL}$ de água destilada e transferido para pesa-filtros para secagem em estufa a $100{ }^{\circ} \mathrm{C}$ até massa constante. A percentagem de lignina insolúvel foi calculada em relação à massa de amostra seca conforme a Equação 1.

$$
\% L_{K i}=\frac{M_{K}-M_{C}}{M_{A}} .100
$$

Onde $L_{K i}$ é a lignina Klason insolúvel, $M_{K}$ é a massa de lignina insolúvel seca, $M_{C}$ é a massa de cinzas e $M_{A}$ é a massa da amostra seca.

A quantidade de lignina solúvel foi determinada pela medida de absorbância a $280 \mathrm{~nm}$ em espectrofotômetro. O cálculo da lignina solúvel foi determinado de acordo com a Equação 2.

$$
C_{\text {lig }}=4,87 \cdot 10^{-2}\left(A_{T}-A_{p d}\right)-3,279 \cdot 10^{-4}(2)
$$

onde $C_{l i g}$ é a concentração de lignina solúvel (g/L), $A_{T}$ é a absorbância $(280 \mathrm{~nm})$ da solução de lignina junto com os produtos de degradação e $A_{p d}=\mathrm{c}_{1} \varepsilon_{1}+\mathrm{c}_{2} \varepsilon_{2}$ é a absorbância $(280 \mathrm{~nm})$ dos produtos de decomposição dos açúcares (furfural e HMF), cujas concentrações $c_{1}$ e $c_{2}$ foram determinadas previamente por CLAE (cromatografia líquida de alta eficiência) e as absortividades, $\varepsilon_{1}$ e $\varepsilon_{2}$, valem, respectivamente, 146,85 e 114,00 L/g.cm

Para a construção das curvas de calibração dos carboidratos, foram injetadas no cromatógrafo líquido soluções contendo celobiose, glicose, xilose e arabinose. A construção das curvas de calibração dos ácidos orgânicos foi realizada através da injeção de soluções contendo ácido acético e ácido fórmico, sendo as análises realizadas em cromatógrafo Shimadzu sob as seguintes condições: coluna Aminex HPX 87H (300 x 7,8 mm, Bio-Rad), fase móvel: $\mathrm{H}_{2} \mathrm{SO}_{4}$ 0,005 mol/ L, vazão 0,6 $\mathrm{mL} / \mathrm{min}$ e temperatura do forno, $45^{\circ} \mathrm{C}$.

Para determinar as concentrações de hidroximetilfurfural (HMF) e furfural foram realizadas análises cromatográficas (CLAE) em cromatógrafo Shimadzu. Construíram-se curvas de calibração de furfural e hidroximetilfurfural injetando soluções padrões destes dois compostos. As amostras dos 
hidrolisados foram filtradas em membrana de $0,45 \mu \mathrm{m}$ e analisadas (Gouveia et al., 2009). As condições experimentais foram: coluna C-18 (Waters, 3,9 x $300 \mathrm{~mm}$ ), fase móvel solução de acetonitrila/água 1:8 com $1 \%$ de solução de ácido acético, vazão $0,8 \mathrm{~mL} / \mathrm{min}$, temperatura do forno de $25^{\circ} \mathrm{C}$ e detector UV/VIS (SPD-10A) a $274 \mathrm{~nm}$.

Após a determinação da lignina insolúvel em meio ácido, a mesma, juntamente com o papel de filtro, foi transferida para um cadinho de porcelana, previamente tarado. A amostra foi calcinada lentamente até $300{ }^{\circ} \mathrm{C}$ e mais $2 \mathrm{~h}$ a $800{ }^{\circ} \mathrm{C}$, em uma mufla. Para determinação das cinzas totais foram coletadas $2 \mathrm{~g}$ da fibra da casca do coco verde para serem incineradas com o mesmo procedimento anterior. A Equação 3 determina o teor de cinzas.

$$
\% \text { Cinzas }=\frac{\text { Massafinal }}{\text { Massainicial }} \cdot 100
$$

\section{Resultados e discussão}

A caracterização da biomassa in natura e pré-tratada, com os valores médios dos constituintes da fibra da casca do coco verde convertidos em celulose e hemicelulose, lignina total e cinzas e extrativos, são apresentados na Tabela 1. Os ensaios foram realizados em duplicata.

Tabela 1. Composição química da fibra da casca do coco verde in natura e após pré-tratamentos.

\begin{tabular}{ccccc}
\hline Componente & in natura & $\begin{array}{c}\text { Aragão } \\
\mathbf{( 2 0 0 7 )}\end{array}$ & $\begin{array}{c}\text { Pré-tratamento } \\
\text { ácido }\end{array}$ & $\begin{array}{c}\text { Pré-tratamento } \\
\text { alcalino }\end{array}$ \\
\hline Celulose (\%) & 24,70 & 23 a 43 & 38,09 & 55,17 \\
Hemicelulose (\%) & 12,26 & 3 a 12 & 1,46 & 7,80 \\
Lignina (\%) & 40,10 & 35 a 45 & 57,70 & 29,91 \\
Cinzas (\%) & 2,56 & - & 2,12 & 0,89 \\
Extrativos (\%) & 2,63 & - & - & - \\
\hline Total & $\mathbf{8 2 , 2 5}$ & - & $\mathbf{9 9 , 3 7}$ & $\mathbf{9 3 , 7 7}$ \\
\hline
\end{tabular}

A quantidade de lignina na composição da fibra da casca do coco foi bastante elevada, com valor médio de $40,10 \%$. Isto explica o alto grau de durabilidade e resistência deste material. Em comparação a outros materiais lignocelulósicos, esta biomassa apresenta quantidade de celulose representativa $(24,70 \%)$, o que lhe oferece boas condições para sua utilização na produção de etanol de segunda geração. O bagaço de cana-de-açúcar, que hoje é o resíduo agroindustrial de maior estudo nesta área, apresenta 46,8\% de celulose, 27,5\% de hemicelulose e $26,2 \%$ de lignina na sua composição (Canilha et al., 2007). Segundo Moraes et al. (2012), a palha da cana-de-açúcar possui cerca de 30\% celulose, mostrando grande potencial para o aproveitamento energético e industrial. Para o milho, Santos (2014) obteve para o sabugo e a palha, 35,4\% e 36,7\% de celulose, respectivamente. 
O pré-tratamento ácido, com maiores rendimentos mássicos, tem nesta biomassa não apenas celulose $(38,09 \%)$ em grande quantidade, como é o caso da biomassa pré-tratada com reagente alcalino, mas também significativo percentual de lignina (57,70\%), que pode vir a interferir no processo de hidrólise enzimática. Uma vantagem do pré-tratamento ácido é a solubilização da hemicelulose, que restou apenas $1,46 \%$ na composição do material pré-tratado. Entretanto, existe o risco de formação de produtos voláteis de degradação do carbono e estes, em muitos casos, diminuem a conversão a etanol (Palmqvist e Hahni, 2000).

O principal efeito do pré-tratamento alcalino consiste na remoção da lignina da biomassa, promovendo maior reatividade da fibra (Baudel, 2006). A proporção de lignina reduziu de 40,10\% no material desidratado para $29,91 \%$ no pré-tratado, proporcionando o aumento na fração de celulose de 24,70 para $55,17 \%$. Em relação à hemicelulose, houve redução de $12,26 \%$ para 7,80\%. Nascimento et al. (2011), trabalhando com bagaço de cana-de-açúcar, conseguiram, na condição de $7 \% \mathrm{NaOH}$ e 30 minutos na deslignificação, reduzir a concentração de lignina para 5\% na biomassa e obter teor de celulose em torno de $38 \%$, garantindo um alto conteúdo acessível de celulose para degradação enzimática.

O percentual de degradação é obtido realizando um balanço de massa dos materiais antes e depois do pré-tratamento (Figura 1). É possível visualizar que a degradação da celulose foi mais acentuada no pré-tratamento alcalino, apresentando quase o dobro de perda em relação ao prétratamento ácido. Apesar da significativa perda, a biomassa tratada com $\mathrm{NaOH}$ apresenta uma solubilização de lignina em torno de $80 \%$, ao contrário do tratamento em meio ácido, que solubilizou apenas $28 \%$. Como já esperado, a solubilização da hemicelulose em meio ácido foi mais efetiva do que em meio básico, apresentando valores de 94 e $80 \%$ de degradação, respectivamente.

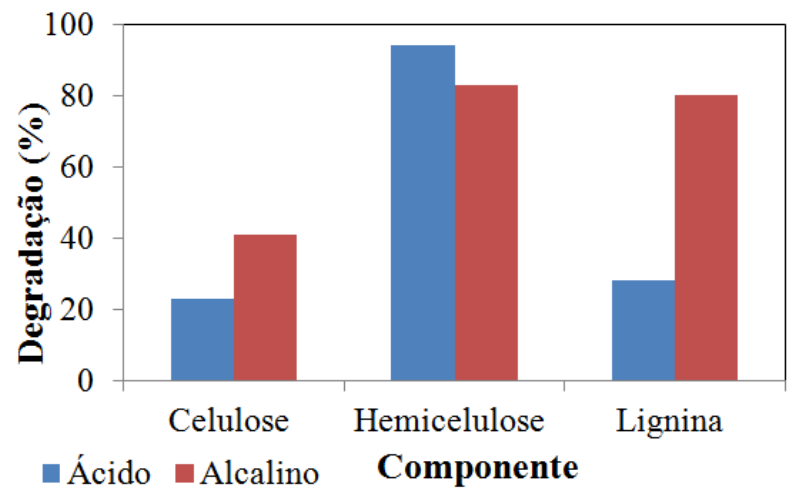

Figura 1. Degradação dos componentes químicos da fibra da casca do coco verde após prétratamento.

Santos (2014) obteve para a palha do milho remoção de $25,1 \%$ no teor de celulose, $70,66 \%$ de hemicelulose e 39,34\% de lignina. Já para o sabugo do milho, houve degradação de 36,6\% de celulose e solubilização de $67,8 \%$ e $28,6 \%$ de hemicelulose e lignina, respectivamente. 
Câmara e Moraes (2012), utilizando peróxido de hidrogênio alcalino para o pré-tratamento do bagaço de cana-de-açúcar, conseguiram remover 51,58\%, 30,75\% e 17,9\% da lignina, hemicelulose e celulose presentes inicialmente, respectivamente, apresentando teores finais de $16,87 \%$ de lignina, $28,5 \%$ de hemicelulose e $48,9 \%$ de celulose.

Considerando que após os pré-tratamentos houve uma perda de massa em torno de 50\% para o ácido e de 75\% para o alcalino, pode-se afirmar que, mesmo com baixo rendimento mássico e maior degradação da cadeia celulósica, o pré-tratamento alcalino apresentou melhores resultados do que o ácido, principalmente pela elevada concentração de celulose na massa pré-tratada e alta solubilização de lignina. No entanto, antes de se excluir esta técnica como melhor forma de tratamento das fibras de coco, são necessários estudos de hidrólise enzimática da biomassa prétratada e de fermentação, avaliando-se o processo como um todo.

\section{Conclusões}

A caracterização química da fibra da casca do coco verde constatou elevada concentração de lignina, com valor médio de $40,10 \%$, justificando o alto grau de durabilidade e resistência deste material, e quantidade de celulose representativa $(24,70 \%)$, oferecendo boas condições para sua utilização na produção de etanol de segunda geração. Em relação aos pré-tratamentos, o alcalino, apesar da significativa perda em celulose, apresentou uma significativa solubilização de lignina (80\%), sendo considerado o mais viável para estudos da produção de etanol 2G.

\section{Agradecimentos}

Os autores agradecem o apoio financeiro do Conselho Nacional de Desenvolvimento Científico e Tecnológico (CNPq) e o auxílio do Departamento de Engenharia Química da Universidade Federal de São Carlos (DEQ/UFSCar) nas análises de caracterização.

\section{Referências bibliográficas}

ARAGÃO, W. M. 2007. Cultivares de coqueiros. In: FONTES, H. R.; FERREIRA, J. M.S., SIQUEIRA, L. A. (Ed.). A cultura do coqueiro. Aracaju: Embrapa Tabuleiros Costeiros. Disponível em:

http://sistemasdeproducao.cnptia.embrapa.br/FontesHTML/Coco/ACulturadoCoqueiro/cultivares.h tm (Acesso 6 Janeiro 2015).

BALAT, M. 2011. Production of bioethanol from lignocellulosic materials via the biochemical pathway: A review. Energy Conversion and Mangement, 52, 2, 858-875.

BARRETO, G. C. 2009. Levantamento das tecnologias para produção de etanol de segunda geração: o potencial do Brasil. Trabalho de Conclusão de Curso - Departamento de Engenharia Química, Universidade Salvador, Salvador, $71 \mathrm{f}$.

BAUDEL, H. M. 2006. Pré-tratamento e hidrólise. III Workshop tecnológico sobre hidrólise para a produção de etanol. Projeto Programa de Pesquisa em Políticas Públicas - Etanol, São Paulo.

BRITO, E. O., ROCHA, J. D. S., VIDAURRE, G. B., BATISTA, D. C., PASSOS, P. R. A., MARQUES, L. G. C. 2004. Propriedades de chapas produzidas com resíduos do fruto de coco e partículas de pinus. Floresta e Ambiente, 11, 2, 01-06. 
CÂMARA, M. M., MORAES, F. F. 2012. Avaliação do pré-tratamento de bagaço de cana por peróxido de hidrogênio alcalino: I. Rendimento mássico e composição química da biomassa. In: VII Encontro de Produção Científica e Tecnológica - EPCT, 2012, Campo Mourão - PR. 22 a 26 de outubro de 2012.

CANILHA, L., CARVALHO, W., ROCHA, G. J. M., ALMEIDA e SILVA, J. B., GIULIETTI, M. 2007. Caracterização do bagaço de cana-de-açúcar in natura, extraído com etanol ou cilohexano/etanol. In: XLVII Congresso Brasileiro de Química (CBQ), Natal/RN. 17 a 21 de setembro de 2007.

CARRIJO, O.A.; LIZ, R.S.; MAKISHIMA, N. 2002. Fibra da casca do coco verde como substrato agrícola. Horticultura Brasileira, Brasília, 20, 4, 533-535.

CÉSAR, S. F., SILVEIRA, M. S., CUNHA, R. D. A. 2010. Aproveitamento de cascas de coco verde para produção de briquete em Salvador - uma alternativa sustentável para a destinação de resíduos do coco in natura. In: José Jéferson do Rêgo Silva; Miguel Aloysio Sattler. (Org.). Sustentabilidade do ambiente construído. O que você tem com isso? 1ed. Recife: ANTAC, 1, 311-321.

CHEMMÉS, C. S., SILVA, F. C., SOUZA, L. S., AZEVEDO JR, R. A., CAMPOS, L. M. A. 2013. Estudo de métodos físico-químicos no pré-tratamento de resíduos lignocelulósicos para produção de etanol de segunda geração. XII SEPA - Seminário Estudantil de Produção Acadêmica, UNIFACS, 12.

COSTA, J. E.; GEBARA, J. J. 2001. A cadeia produtiva do coco em Sergipe. In: GERARDI, L. H. O. (org.) Teoria, técnica, espaços e atividades: temas de geografia contemporânea. Rio Claro: UNESP, São Paulo.

GOUVEIA, E. R.; NASCIMENTO, R. T.; SOUTO-MAIOR, A. M. 2009. Validação de metodologia para a caracterização química do bagaço de cana de açúcar. Química Nova, 32, 6, 500-1503.

MACIEL, T. C.,OLIVEIRA, S. L. R. ; RODRIGUES, S. 2010. Investigação do potencial produtor de celulases de fungos isolados da casca do coco verde (Cocos nucifera $\mathrm{L}$.) e de Trichoderma reesei NRRL 60 utilizando resíduo agroindustrial como substrato. In: I Congresso do Instituto Nacional de Frutos Tropicais e II Simpósio em Ciência e Tecnologia de Alimentos, Aracaju.

MARTINS, C. R., JESUS JR, L. A. 2013. Produção e comercialização de coco no Brasil frente ao comércio internacional: panorama 2014. Embrapa Tabuleiros Costeiros, 51 p. (Documentos/Embrapa Tabuleiros Costeiros, ISSN 1517-1329; 184).

MORAES, M. S. A., GEORGES, F., RODRIGUES, S. A., DAMASCENDO, F. C., MACIEL, G. P. S., ZINI, C. A., JACQUES, R. A., CARAMÃO, E. B. 2012. Analysis of products from pyrolysis of Brazilian sugar cane straw. Fuel Processing Technology, 93, 35-43.

NASCIMENTO, V. M., ROCHA, G. J. M., GIORDANO, R. C., CRUZ, A. J. G. 2011. Optimization of alkaline $(\mathrm{NaOH})$ pretreatment of sugarcane bagasse for production of second generation bioethanol. In: Society for Industrial Microbiology.

PALMQVIST, E., HAHNl, B. 2000. Fermentation of lignocellulosic hydrolysates. II: Inhibitors and mechanisms of inhibition. Bioresource Technology, 74, 25-33.

PEREIRA, C. L., SAVASTANO, H., PAYÁ, J., SANTOS, S.F., BORRACHERO, M.V., MONZÓ, J., SORIANO, L. 2013.Use of highly reactive rice husk ash in the production of cement matrix reinforced with green coconut fiber. Industrial Crops and Products, 49, 88-96.

PIMENTA, A. S., SANTOS, R. C., CARNEIRO, A. C. O., CASTRO, R. V. O. 2015. Utilização de resíduos de coco (Cocos nucifera) carbonizado para a produção de briquetes. Ciência Florestal. Santa Maria, 25, 1, 137-144.

ROSA, M. F., ABREU, F. A. P., FURTADO, A. A. L., BRÍGIDO, A. K. L., NORÕES, E. R. V. 2001. Processo agroindustrial: obtenção de pó de casca de coco verde. Fortaleza: Embrapa Agroindústria Tropical (Comunicado Técnico, 61).

SAMPAIO, A. R., RAMOS, S. J., GUILHERME, D. O., COSTA, C. A., FERNANDES, L. A. 2008. Produção de mudas de tomateiro em substratos contendo fibra de coco e pó de rocha. Horticultura Brasileira, 26, 499-503.

SANTOS, M. S. R. 2014.Estudo de pré - tratamentos de palha e sabugo de milho visando a produção de etanol 2G. Dissertação (Mestrado) - Programa de pós graduação em engenharia química Universidade Federal de Alagoas, Alagoas, Maceió.75 p. 
SARKAR, N., GHOSH, S. K., BANNERJEE, S., AIKAT, K. 2012. Bioethanol production from agricultural wastes: an overview. Renewable Energy, 1, 19-27.

SENHORAS, E. M. 2003. Estratégias de uma agenda para a cadeia agroindustrial de coco. Monografia, Instituto de Economia, UNICAMP, Campinas. 38 p.

SENHORAS, E. M. 2004. Oportunidades da cadeia agroindustrial do coco verde: do coco verde nada se perde, tudo se desfruta. Portal Urutágua. Disponível em: http://www.urutagua.uem.br/005/22eco_senhoras.htm\#_ftn1 (Acesso 02 Janeiro 2014).

SILVA, K. M. D., REZENDE, L. C. S. H., SILVA, C. A., BERGAMASCO, R., GONÇALVES, D. S. 2013. Caracterização físico-química da fibra de coco verde para a adsorção de metais pesados em efluente de indústria de tintas. ENGEVISTA, 5, 1, 43-50. 